\title{
Portfolio Optimization Model with and without Options under Additional Constraints
}

\author{
T. Khodamoradi, ${ }^{1}$ M. Salahi $(1),{ }^{1,2}$ and Ali Reza Najafi ${ }^{1}$ \\ ${ }^{1}$ Department of Applied Mathematics, Faculty of Mathematical Sciences, University of Guilan, Rasht, Iran \\ ${ }^{2}$ Center of Excellence for Mathematical Modeling, Optimization and Combinatorial Computing (MMOCC), \\ University of Guilan, Rasht, Iran
}

Correspondence should be addressed to M. Salahi; salahi.maziar@gmail.com

Received 31 August 2020; Revised 5 October 2020; Accepted 14 October 2020; Published 26 November 2020

Academic Editor: S. A. Edalatpanah

Copyright (c) 2020 T. Khodamoradi et al. This is an open access article distributed under the Creative Commons Attribution License, which permits unrestricted use, distribution, and reproduction in any medium, provided the original work is properly cited.

\begin{abstract}
In this paper, first, we study mean-absolute deviation (MAD) portfolio optimization model with cardinality constraints, short selling, and risk-neutral interest rate. Then, in order to insure the investment against unfavorable outcomes, an extension of MAD model that includes options is considered. Moreover, since the data in financial models usually involve uncertainties, we apply robust optimization to the MAD model with options. Finally, a data set of S\&P index is used to compare the effectiveness of options in the models in terms of returns and Sharpe ratios.
\end{abstract}

\section{Introduction}

The mean-variance (MV) model proposed by Markowitz [1] is a single-period model that provides the best trade-off between return and risk. It is a quadratic programming problem; so, when the number of stocks is large, estimating the covariance matrix could be difficult. Then, Konno and Yamazaki [2] proposed the mean-absolute deviation (MAD) model with the absolute deviation of the rate of portfolio return as a measure of the risk instead of the variance. They proved that the MAD model gives essentially the same results as the MV model if all the returns are normally distributed random variables. Feinstein and Thapa [3] reformulated the MAD model with constraints less than the model of Konno and Yamazaki. Later, Chang [4] provided a reformulation of the model proposed by Feinstein and Thapa. Gorard [5] presented a review of the MAD versus the standard deviation. Kasenbacher et al. [6] also compared the MV model and the MAD model. Further studies on the MAD model can be found in [7-16].

Some extensions of the MAD model include short selling, threshold, and cardinality constraints. Short selling is the sale of a stock that does not belong to the seller. Investors under it borrow the stock to repay it in the future when they believe that the price of the stock will decline. After a while, the seller buys the stock from the market and repays it to the lender. Lintner [17] studied the first model of short selling in the portfolio theory. Konno et al. used the MAD model with the long-short strategy and showed that the long-short strategy leads to a portfolio with significantly better risk-return structure compared to the portfolio with the long strategy.

Cardinality constraints put a limit on the stock number in the portfolio, and the constraints of the threshold restrict the weights of stock in the portfolio to lie between given lower bounds and upper bounds. If the portfolio selects a small number of stocks from a large investment space, it means sparse [18-20]. Kwon and Stoyan [21] used the MAD model with cardinality constraints. They solved the MV and MAD models with different trading constraints and observed that the MAD model is substantially more tractable. In 2014, Le Thi and Moeini [22] extended the MAD model with short selling, cardinality, and the threshold constraints. Their model is reformulated in terms of a DC (difference of convex functions) problem and applied DC algorithms to solve it [23-25]. Cardinality constraints are also discussed in the MV models, such as the works of Anagnostopoulos and Mamanis [26], Gao and Li [27], Cesarone et al. [28], and Salahi et al. [29, 30]. 
Another factor that can be used in the portfolio optimization model is option. It is a financial derivative that can be considered as an asset for investment [31] and expresses as a contract that gives the holder the right to exercise a deal, but the contractor is not obliged to accomplish this right [32]. A call or put option gives the contractor the right to buy or sell the underlying stock at a certain price over a specified time. European or American options are the most common options that differ in the period of exercising the option. In European option, a contractor can only be applied the option at the expiration time, and in American option, a contractor should decide whether or not to exercise the option in any time before or at the expiration time [33]. In 2011, Topaloglou et al. [34] studied the options in the single-period portfolio. They found that controlling the risk of the market with options had a significant effect on performance of portfolio relative to currency risk. Authors in [35] showed that option reduces the risk and leads to better portfolio performance. Other studies also have investigated portfolio optimization models with options, for example, see [36-42].

Since the future of the financial market is ambiguous, historical data play a key role in predicting the future of the market. The stock returns forecasting is significant for stock pricing, stock allocation, and risk management. Dai et al. [43] improved the accuracy of stock return forecasts by combining a new two-step economic constraint forecasting model and new technical indicators. Also Dai et al. [44] found that combining denoising of stock returns with wavelet transform with new technical indicators can significantly improve the accuracy of stock returns forecasting, where the new technical indicators can directly reflect the trend of stock returns series. However, along with all the advantages of these forecasting methods, they can lead to some errors. On the other hand, the solutions of optimization problems show significant sensitivity to perturbations in the input parameters. A small uncertainty in the input parameters can make the usual optimal solution practically meaningless. Then, there is a need to develop models that are as safe as possible to the data uncertainty. Robust optimization is one of the widely used approaches to deal with uncertainties. In this approach, uncertain parameters are considered within known sets that are called uncertainty sets. First, Soyster [45] studied robust counterpart optimization using interval uncertainty sets. Then, Ben-Tal and Nemirovski [46] suggested that the ellipsoid uncertainty set and the robust formulation become a conic quadratic optimization problem. Although the proposed model is less conservative than Soyster's approach, the problem is nonlinear. Further, Bertsimas and Sim [47] studied robust linear optimization with coefficient uncertainty using an uncertainty set with budgets where their model is less conservative and stays linear. Moon and Yao [48] studied the robust MAD model. Their model led to a linear programming problem and reduced computational burden of the earlier robust portfolio optimization models. Lutgens et al. [39] studied a robust optimization for option hedging problems under ellipsoidal uncertainty sets. Their model is formulated as a second-order cone problem. Zymler et al. [49] developed a robust optimization model for designing portfolio including
European options that trades off strong and weak guarantees on the worst-case portfolio return. Further important studies in the subject can be found in [50-60].

The goal of this paper is to analyze the MAD model with and without options when short selling, risk-neutral interest rate, and cardinality constraints are allowed. Then, the robust formulation under the interval uncertainty sets is studied. The rest of this paper is structured as follows. In Section 2, we describe the MAD model with short selling, risk-neutral interest rate, and cardinality constraints in detail. In Section 3, we extend the MAD model to include options. Robust model under interval uncertainties is discussed in Section 4. Numerical results are given in Section 5. Finally, Section 6 concludes the paper.

\section{MAD Model and Extensions}

The MAD model is as follows [2]:

$$
\begin{aligned}
& \min _{x, u} \lambda\left(\frac{1}{T} \sum_{t=1}^{T}\left|\sum_{j=1}^{N}\left(r_{t j}-r_{j}\right) x_{j}\right|\right)-(1-\lambda)\left(\sum_{j=1}^{N} r_{j} x_{j}\right) \\
& \text { s.t. } \quad \sum_{j=1}^{N} x_{j}=1, \\
& \varepsilon_{j} \leq x_{j} \leq \delta_{j}, j=1, \ldots, N \\
& x_{j} \geq 0, j=1, \ldots, N,
\end{aligned}
$$

where $T$ and $N$ denote the end of investment time and the number of available stocks, respectively, $r_{t j}$ is the return of the $j$ th stock at time $t,(t=1, \ldots, T ; j=1, \ldots, N)$, and $r_{j}=$ $(1 / T) \sum_{t=1}^{T} r_{t j}$ for the $j$ th stock $(j=1, \ldots, N)$. Also, $x_{j}$ is the weight of $j$ th stock, $\varepsilon_{j}$ and $\delta_{j}$ represent the lower bound and upper bound of the $j$ th stock, respectively, and $\lambda \in[0,1]$ is the risk aversion parameter.

To include realistic constraints in the MAD model, by adding short selling, risk-neutral interest rate, and cardinality constraints, we get the following model [29]:

$$
\begin{gathered}
\min _{x, z} \lambda\left(\frac{1}{T} \sum_{t=1}^{T}\left|\sum_{j=1}^{N}\left(r_{t j}-r_{j}\right) x_{j}\right|\right) \\
\quad-(1-\lambda)\left(\sum_{j=1}^{N}\left(r_{j} x_{j}-r_{c} h_{j} x_{j}\right)\right) \\
\text { s.t. } \quad \sum_{j=1}^{N} x_{j}=1, \\
\quad \sum_{j=1}^{N} z_{j}=K, \\
r_{j} x_{j} \geq 0, j=1, \ldots, N, \\
\varepsilon_{j} z_{j} \leq x_{j} \leq \delta_{j} z_{j}, j=1, \ldots, N, \\
z_{j} \in\{0,1\}, j=1, \ldots, N,
\end{gathered}
$$


where $r_{c}$ is risk-neutral interest rate, $\mathrm{K}$ is the number of stocks in the portfolio, and $z_{j}$ 's are binary variables. If $z_{j}=1$, stock $j$ belongs to the portfolio, and if $z_{j}=0$, it does not. The term $r_{c} \sum_{j=1}^{N} h_{j} x_{j}$ represents the short rebate, where

$$
0<h_{j}<1, \quad \forall j
$$

is the investor's portion of the interest received on proceeding from the short sale of stock $j$. Then, $h_{j}=0$ when $r_{j} \geq 0$ and $0<h_{j}<1$ when $r_{j}<0$. The constraints $r_{j} x_{j} \geq 0(j=1, \ldots, N)$ show that, for any stock which is in the short selling position, the proportion of investment is negative. The objective function in model (2) is nonlinear; however, using auxiliary variable $u_{t}$, we get the following linear model:

$$
\begin{array}{ll}
\min _{x, z, u} \lambda\left(\frac{1}{T} \sum_{t=1}^{T} u_{t}\right)-(1-\lambda)\left(\sum_{j=1}^{N}\left(r_{j} x_{j}-r_{c} h_{j} x_{j}\right)\right) \\
\text { s.t. } \quad u_{t}+\sum_{j=1}^{N}\left(r_{t j}-r_{j}\right) x_{j} \geq 0, t=1, \ldots, T, \\
\\
u_{t}-\sum_{j=1}^{N}\left(r_{t j}-r_{j}\right) x_{j} \geq 0, t=1, \ldots, T, \\
\sum_{j=1}^{N} x_{j}=1, \\
\sum_{j=1}^{N} z_{j}=K, \\
u_{t} \geq 0, t=1, \ldots, T, \\
r_{j} x_{j} \geq 0, j=1, \ldots, N, \\
\varepsilon_{j} z_{j} \leq x_{j} \leq \delta_{j} z_{j}, j=1, \ldots, N, \\
z_{j} \in\{0,1\}, j=1, \ldots, N .
\end{array}
$$

It should be noted that, $\varepsilon_{j}$ is negative when short selling is allowed.

\section{MAD Model with Options}

In this section, we use options in the portfolio that ensure the investment against unfavorable outcomes. They reduce the risk and come, however, at some costs that decrease the return of the portfolio [35]. These costs (options prices) are formulated based on the risk-neutral interest rate as follows:

$$
\begin{aligned}
& O_{\text {put }}=\max \left\{0, K-S_{T}\right\} e^{-r_{c} T}, \\
& O_{\text {call }}=\max \left\{0, S_{T}-K\right\} e^{-r_{c} T},
\end{aligned}
$$

where $S_{T}$ is the stock price vector in the expiration time and $K$ is strike price such that

$$
K=S_{0} e^{-r_{c} T},
$$

where $S_{0}$ is a vector of stock initial price. Since we use $O_{\text {call }}$ and $O_{\text {put }}$ for any stock, the total option price is

$$
O=O_{\text {call }}+O_{\text {put }}
$$

Using these call and put options under strike price $(K)$, the option payoff functions become

$$
\begin{aligned}
& V_{\text {put }}\left(S_{T}\right)=\max \left\{0, K-S_{T}\right\}, \\
& V_{\text {call }}\left(S_{T}\right)=\max \left\{0, S_{T}-K\right\} .
\end{aligned}
$$

Based on these payoff functions, options returns are as follows:

$$
\begin{aligned}
& R_{\text {put }}=\frac{1}{S_{0}} \max \left\{0, K-S_{T}\right\}, \\
& R_{\text {call }}=\frac{1}{S_{0}} \max \left\{0, S_{T}-K\right\} .
\end{aligned}
$$

Using $S_{T}$ and $K$, the investor decides whether to exercise the call or put options for any stock. Therefore, model (4) under the options returns and options prices becomes

$$
\begin{aligned}
& \min _{x, z, u} \lambda\left(\frac{1}{T} \sum_{t=1}^{T} u_{t}\right)-(1-\lambda)\left(\sum_{j=1}^{N}\left(r_{j} x_{j}-r_{c} h_{j} x_{j}\right)\right. \\
& \left.-\sum_{j=1}^{N} O_{j}\left|x_{j}\right|+\sum_{j=1}^{N}\left(\left(R_{\text {call }}\right)_{j}+\left(R_{\text {put }}\right)_{j}\right) x_{j}\right) \\
& \text { s.t. } u_{t}+\sum_{j=1}^{N}\left(r_{t j}-r_{j}\right) x_{j} \geq 0, t=1, \ldots, T \text {, } \\
& u_{t}-\sum_{j=1}^{N}\left(r_{t j}-r_{j}\right) x_{j} \geq 0, t=1, \ldots, T, \\
& \sum_{j=1}^{N} x_{j}=1 \\
& \sum_{j=1}^{N} z_{j}=K \\
& u_{t} \geq 0, t=1, \ldots, T \text {, } \\
& r_{j} x_{j} \geq 0, j=1, \ldots, N \text {, } \\
& \varepsilon_{j} z_{j} \leq x_{j} \leq \delta_{j} z_{j}, j=1, \ldots, N \text {, } \\
& z_{j} \in\{0,1\}, j=1, \ldots, N,
\end{aligned}
$$

which is a mixed-integer linear optimization problem. In this model, since short selling is allowed, the returns of options for stocks in these situations are considered negative. 
Lemma 1. Let $Z^{*}$ and $W^{*}$ denote the optimal objective values of optimization models (4) and (10), respectively. Then, $Z^{*} \geq W^{*}$.

Proof. Let $\left(x^{*}, u^{*}\right)$ be an optimal solution of model (4). We have

$$
\begin{aligned}
\frac{\lambda}{T} \sum_{t=1}^{T} u_{t}^{*}-(1-\lambda)\left(\sum_{j=1}^{N}\left(r_{j} x_{j}^{*}-r_{c} h_{j} x_{j}^{*}\right)\right) \\
\geq \frac{\lambda}{T} \sum_{t=1}^{T} u_{t}^{*}-(1-\lambda)\left(\sum_{j=1}^{N}\left(r_{j} x_{j}^{*}-r_{c} h_{j} x_{j}^{*}\right)-\sum_{j=1}^{N} O_{j}\left|x_{j}^{*}\right|\right. \\
\left.\quad+\sum_{j=1}^{N}\left(\left(R_{\text {call }}\right)_{j}+\left(R_{\text {put }}\right)_{j}\right) x_{j}^{*}\right),
\end{aligned}
$$

since

$$
0 \leq O_{j} \leq\left(R_{\text {call }}\right)_{j}+\left(R_{\text {put }}\right)_{j} .
$$

Therefore, $Z^{*} \geq W^{*}$.

\section{Robust Model}

In this section, since the future values of stock prices may involve uncertainties, we use robust optimization to deal with this situation. In this approach, input parameters are considered in bounded uncertainty sets that contain all or most values of uncertain data. Model (10) under uncertainty is

$$
\begin{aligned}
& \min _{x, z, u, r} \lambda\left(\frac{1}{T} \sum_{t=1}^{T} u_{t}\right)-(1-\lambda)\left(\sum_{j=1}^{N}\left(\widetilde{r}_{j} x_{j}-r_{c} h_{j} x_{j}\right)-\sum_{j=1}^{N} O_{j}\left|x_{j}\right|+\sum_{j=1}^{N}\left(\left(R_{\text {call }}\right)_{j}+\left(R_{\text {put }}\right)_{j}\right) x_{j}\right) \\
& \text { s.t. } \quad u_{t}+\sum_{j=1}^{N}\left(\widetilde{r}_{t j}-\widetilde{r}_{j}\right) x_{j} \geq 0, t=1, \ldots, T, \\
& \qquad u_{t}-\sum_{j=1}^{N}\left(\widetilde{r}_{t j}-\widetilde{r}_{j}\right) x_{j} \geq 0, t=1, \ldots, T, \\
& \quad \sum_{j=1}^{N} x_{j}=1, \\
& \quad \sum_{j=1}^{N} z_{j}=K, \\
& \quad z_{j} \in\{0,1\}, j=1, \ldots, N, \\
& \tilde{r}_{j} \in U_{1}, j=1, \ldots, N \\
& \tilde{r}_{t j} \in U_{2}, t=1, \ldots, T, j=1, \ldots, N, \\
& \tilde{r}_{j} x_{j} \geq 0, j=1, \ldots, N, \\
& z_{j} \leq x_{j} \leq \delta_{j} z_{j}, j=1, \ldots, N,
\end{aligned}
$$


where

$$
\begin{aligned}
& U_{1}=\left\{\widetilde{r}_{j} \mid r_{j}^{l} \leq \widetilde{r}_{j} \leq r_{j}^{u}, \quad j=1, \ldots, N\right\}, \\
& U_{2}=\left\{\widetilde{r}_{t j} \mid r_{t j}^{l} \leq \widetilde{r}_{t j} \leq r_{t j}^{u}, \quad t=1, \ldots, T, j=1, \ldots, N\right\} .
\end{aligned}
$$

Here, $r_{t j}^{l}$ and $r_{t j}^{u}$ denote the lower bound and upper bound of returns for stock $j$ at time $t$, respectively. Also, $r_{j}^{l}$ and $r_{j}^{u}$ denote the lower bound and upper bound of $r_{j}$, respectively.

Theorem 1. The robust counterpart of model (13) for uncertainty sets (14) is as follows:

$$
\begin{aligned}
& \min _{x, z, u, r} \lambda\left(\frac{1}{T} \sum_{t=1}^{T} u_{t}\right)-(1-\lambda)\left(\sum_{j=1}^{N}\left(-r_{j}^{u} s_{j}+r_{j}^{l} c_{j}\right)-\sum_{j=1}^{N} r_{c} h_{j} x_{j}-\sum_{j=1}^{N} O_{j}\left|x_{j}\right|+\sum_{j=1}^{N}\left(\left(R_{\text {call }}\right)_{j}+\left(R_{\text {put }}\right)_{j}\right) x_{j}\right) \\
& \text { s.t. } \quad u_{t}+\sum_{j=1}^{N}\left(-r_{t j}^{u} \gamma_{t j}+r_{t j}^{l} \delta_{t j}-r_{j}^{u} \psi_{j}+r_{j}^{l} \varphi_{j}\right) \geq 0, t=1, \ldots, T \text {, } \\
& u_{t}+\sum_{j=1}^{N}\left(-r_{t j}^{u} \alpha_{t j}+r_{t j}^{l} \beta_{t j}-r_{j}^{u} \eta_{j}+r_{j}^{l} \kappa_{j}\right) \geq 0, t=1, \ldots, T \\
& \sum_{j=1}^{N} x_{j}=1 \\
& \sum_{j=1}^{N} z_{j}=K, \\
& u_{t} \geq 0, t=1, \ldots, T \\
& -r_{j}^{u} d_{j}+r_{j}^{l} e_{j} \geq 0, j=1, \ldots, N, \\
& \varepsilon_{j} z_{j} \leq x_{j} \leq \delta_{j} z_{j}, j=1, \ldots, N \\
& s_{j}-c_{j}=-x_{j}, j=1, \ldots, N \text {, } \\
& -\gamma_{t j}+\delta_{t j}=x_{j}, t=1, \ldots, T, j=1, \ldots, N, \\
& -\psi_{j}+\varphi_{j}=-x_{j}, j=1, \ldots, N \\
& -\alpha_{t j}+\beta_{t j}=-x_{j}, t=1, \ldots, T, j=1, \ldots, N \\
& -\eta_{j}+\kappa_{j}=x_{j}, j=1, \ldots, N \text {, } \\
& -d_{j}+e_{j}=x_{j} \\
& s_{j} \geq 0, c_{j} \geq 0, d_{j} \geq 0, e_{j} \geq 0, \psi_{j} \geq 0, \eta_{j} \geq 0, \kappa_{j} \geq 0, j=1, \ldots, N, \\
& \alpha_{t j} \geq 0, \beta_{t j} \geq 0, \gamma_{t j} \geq 0, \delta_{t j} \geq 0, t=1, \ldots, T, j=1, \ldots, N \\
& z_{j} \in\{0,1\}, j=1, \ldots, N \text {. }
\end{aligned}
$$


Proof. The robust counterpart of model (13) under uncertainty sets (14) is

$$
\begin{aligned}
& \min _{x, z, u, r} \lambda\left(\frac{1}{T} \sum_{t=1}^{T} u_{t}\right)+(1-\lambda)\left(\underset{\tilde{r}_{j} \in U_{1}}{\max }\left(-\sum_{j=1}^{N} \widetilde{r}_{j} x_{j}\right)+\sum_{j=1}^{N} r_{c} h_{j} x_{j}+\sum_{j=1}^{N} O_{j}\left|x_{j}\right|\right. \\
& \left.-\sum_{j=1}^{N}\left(\left(R_{\text {call }}\right)_{j}+\left(R_{\text {put }}\right)_{j}\right) x_{j}\right) \\
& \text { s.t. } \quad u_{t}+\min _{\widetilde{r}_{j} \in U_{1}, \widetilde{r}_{t j} \in U_{2}}\left(\sum_{j=1}^{N}\left(\widetilde{r}_{t j}-\widetilde{r}_{j}\right) x_{j}\right) \geq 0, t=1, \ldots, T \text {, } \\
& u_{t}+\min _{\widetilde{r}_{j} \in U_{1}, \widetilde{r}_{t j} \in U_{2}}\left(-\sum_{j=1}^{N}\left(\widetilde{r}_{t j}-\widetilde{r}_{j}\right) x_{j}\right) \geq 0, t=1, \ldots, T, \\
& \sum_{j=1}^{N} x_{j}=1 \\
& \sum_{j=1}^{N} z_{j}=K \\
& u_{t} \geq 0, t=1, \ldots, T \text {, } \\
& \min _{\widetilde{r}_{j} \in U_{1}} \widetilde{r}_{j} x_{j} \geq 0, j=1, \ldots, N \text {, } \\
& \varepsilon_{j} z_{j} \leq x_{j} \leq \delta_{j} z_{j}, j=1, \ldots, N, \\
& z_{j} \in\{0,1\}, j=1, \ldots, N \text {. }
\end{aligned}
$$

In order to simplify model (16), we need equivalent forms of inner maximization and minimization problems. Consider the inner maximization problem in the objective function:

$$
\begin{aligned}
& \max \sum_{j=1}^{N}-\widetilde{r}_{j} x_{j} \\
& \text { s.t. } \quad r_{j}^{l} \leq \widetilde{r}_{j} \leq r_{j}^{u}, j=1, \ldots, N .
\end{aligned}
$$

Its dual is

$$
\begin{gathered}
\min _{s, c} \sum_{j=1}^{N}\left(r_{j}^{u} s_{j}-r_{j}^{l} c_{j}\right) \\
\text { s.t. } \quad s_{j}-c_{j}=-x_{j}, j=1, \ldots, N, \\
\quad s_{j} \geq 0, j=1, \ldots, N, \\
\quad c_{j} \geq 0, j=1, \ldots, N .
\end{gathered}
$$

Since the primal and dual are feasible and the duality gap is equal to zero, we can replace the maximization problem with its dual in the objective function and add its constraints to the model. Now, consider the minimization problem in the first constraint:

$$
\begin{array}{ll}
\min & \sum_{j=1}^{N}\left(\tilde{r}_{t j}-\tilde{r}_{j}\right) x_{j} \\
\text { s.t. } \quad & r_{j}^{l} \leq \widetilde{r}_{j} \leq r_{j}^{u}, j=1, \ldots, N, \\
& r_{t j}^{l} \leq \widetilde{r}_{t j} \leq r_{t j}^{u}, j=1, \ldots, N .
\end{array}
$$

Its dual is

$$
\begin{array}{ll}
\max _{\gamma, \delta, \psi, \varphi} & \sum_{j=1}^{N}\left(-r_{t j}^{u} \gamma_{t j}+r_{t j}^{l} \delta_{t j}-r_{j}^{u} \psi_{j}+r_{j}^{l} \varphi_{j}\right) \\
\text { s.t. } \quad & -\gamma_{t j}+\delta_{t j}=x_{j}, j=1, \ldots, N, \\
& -\psi_{j}+\varphi_{j}=-x_{j}, j=1, \ldots, N, \\
& \gamma_{t j} \geq 0, j=1, \ldots, N, \\
& \delta_{t j} \geq 0, j=1, \ldots, N, \\
& \psi_{j} \geq 0, j=1, \ldots, N, \\
& \varphi_{j} \geq 0, j=1, \ldots, N .
\end{array}
$$

The minimization problem in the second constraint is 
TABLE 1: Companies and their monthly returns for 2016-2018.

\begin{tabular}{|c|c|c|c|}
\hline Company & Return & Company & Return \\
\hline The Bank of New York Mellon Corporation & 0.0113 & Pfizer inc & 0.0089 \\
\hline Electronic Arts Inc. & 0.0146 & Philip Morris International inc & -0.0071 \\
\hline General Dynamics Corporation & 0.0074 & Qualcomm Incorporated & 0.0133 \\
\hline Mylan N.V & -0.0064 & Regeneron Pharmaceuticals Inc. & -0.0074 \\
\hline Newmont Corporation & 0.0227 & Raytheon Technologies Corporation & 0.0079 \\
\hline Truist Financial Corporation & 0.0101 & AT\&T Inc. & 0.0003 \\
\hline Ulta Beauty Inc. & 0.0099 & T-Mobile US Inc. & 0.0177 \\
\hline Dentsply Sirona Inc. & -0.0103 & United Parcel Service Inc. & 0.0044 \\
\hline Booking Holdings inc. & 0.0137 & Verizon Communications Inc. & 0.0084 \\
\hline BlackRock Inc. & 0.0116 & Wells Fargo and Company & 0.0026 \\
\hline Bristol Myers Squibb company & -0.0012 & Exxon Mobil Corporation & -0.0007 \\
\hline Berkshire Hathaway Inc. & 0.0167 & Apache Corporation & -0.0034 \\
\hline Citigroup Inc. & 0.0090 & Comerica Incorporated & 0.0224 \\
\hline Colgate-Palmolive Company & -0.0015 & Hanesbrands Inc. & -0.0121 \\
\hline Comcast Corporation & 0.0121 & Zions Bancorporation, National Association & 0.0217 \\
\hline Virgin Galactic Holdings Inc. & -0.0051 & People's United Financial Inc. & 0.0027 \\
\hline Chevron Corporation & 0.0108 & National Oilwell Varco Inc. & 0.0007 \\
\hline Dominion Energy Inc. & 0.0032 & Marathon Oil Corporation & 0.0162 \\
\hline The Walt Disney Company & 0.0056 & Devon Energy Corporation & 0.0008 \\
\hline Duke Energy Corporation & 0.0046 & Virgin Galactic Holdings Inc. & 0.0085 \\
\hline Gilead Sciences Inc. & -0.0053 & Invesco Ltd. & -0.0090 \\
\hline The Goldman Sachs Group Inc. & 0.0093 & Unum Group & 0.0116 \\
\hline International Business Machines Corporation & -0.0003 & SL Green Realty Corp. & -0.0064 \\
\hline Johnson and Johnson & 0.0063 & Virgin Galactic Holdings Inc. & 0.0139 \\
\hline The Coca-Cola company & 0.0018 & Discovery Inc. & 0.0064 \\
\hline Mondelez International Inc. & -0.0004 & Ralph Lauren Corporation & 0.0102 \\
\hline Altria Group, inc & -0.0053 & The Gap Inc. & 0.0051 \\
\hline Morgan Stanley & 0.0169 & TechnipFMC plc. & -0.0023 \\
\hline Oracle Corporation & 0.0140 & & \\
\hline
\end{tabular}

$$
\begin{array}{ll}
\min & -\sum_{j=1}^{N}\left(\tilde{r}_{t j}-\tilde{r}_{j}\right) x_{j} \\
\text { s.t. } \quad & r_{j}^{l} \leq \widetilde{r}_{j} \leq r_{j}^{u}, j=1, \ldots, N, \\
& r_{t j}^{l} \leq \widetilde{r}_{t j} \leq r_{t j}^{u}, j=1, \ldots, N,
\end{array}
$$

and its dual problem also is

$$
\begin{array}{ll}
\max _{\alpha, \beta, \eta, \kappa} & \sum_{j=1}^{N}\left(-r_{t j}^{u} \alpha_{t j}+r_{t j}^{l} \beta_{t j}-r_{j}^{u} \eta_{j}+r_{j}^{l} \kappa_{j}\right) \\
\text { s.t. } \quad & -\alpha_{t j}+\beta_{t j}=-x_{j}, j=1, \ldots, N, \\
& -\eta_{j}+\kappa_{j}=x_{j}, j=1, \ldots, N, \\
& \alpha_{t j} \geq 0, j=1, \ldots, N, \\
& \beta_{t j} \geq 0, j=1, \ldots, N, \\
& \eta_{j} \geq 0, j=1, \ldots, N, \\
& \kappa_{j} \geq 0, j=1, \ldots, N .
\end{array}
$$

$$
\begin{array}{cl}
\max _{d, e}-r_{j}^{u} d_{j}+r_{j}^{l} e_{j} \\
\text { s.t. } \quad-d_{j}+e_{j}=x_{j}, \\
d_{j} \geq 0, \\
e_{j} \geq 0 .
\end{array}
$$

By replacing the objective functions of dual problems in constraints and adding their constraints to the model, we get the results.

\section{Numerical Experiments}

In this section, we investigate the performance of models (4), (10), and (15). We assume that there are call and put European options on all stocks, and the expiration times of all options are the end of investment times $(T)$. We provide numerical results for the S\&P 5001 index for 2016-2018 when $N=57, \lambda=0.5$, $r_{c}=0.03$, and taking $\delta_{i}=-\varepsilon_{i}=0.1$ as the lower bound and upper bound of the proportion of investment in any stock. The monthly returns of stocks are presented in Table 1 .

For the sake of simplicity, in the robust model, the uncertainty sets are defined as

$$
\begin{aligned}
& U_{1}=\left\{\widetilde{r}_{j} \mid r_{j}-\varepsilon \leq \widetilde{r}_{j} \leq r_{j}+\varepsilon, \quad j=1, \ldots, N\right\} . \\
& U_{2}=\left\{\widetilde{r}_{t j} \mid r_{t j}-\varepsilon \leq \widetilde{r}_{t j} \leq r_{t j}+\varepsilon, \quad t=1, \ldots, T, j=1, \ldots, N\right\},
\end{aligned}
$$

and its dual is

$$
\begin{aligned}
& \min \tilde{r}_{j} x_{j} \\
& \text { s.t. } \quad r_{j}^{l} \leq \widetilde{r}_{j} \leq r_{j}^{u},
\end{aligned}
$$


TABle 2: Comparison of returns of models (4), (10), and (15) with the data of S\&P index data, with $N=57$ stocks for 2016-2018 when $\lambda=0.5$.

\begin{tabular}{lccc}
\hline Number of stocks & Model (4) & Model (10) & Model (15) \\
\hline$K=10$ & 0.0135 & 0.0282 & 0.0214 \\
$K=20$ & 0.0227 & 0.0500 & 0.0206 \\
$K=30$ & 0.0309 & 0.0666 & 0.0291 \\
$K=40$ & 0.0367 & 0.0773 & 0.0297 \\
\hline
\end{tabular}

TABle 3: Comparison of Sharpe ratios of models (4), (10), and (15) with the data of S\&P index data, with $N=57$ stocks for 2016-2018 when $\lambda=0.5$.

\begin{tabular}{lccc}
\hline Number of stocks & Model (4) & Model (10) & Model (15) \\
\hline$K=10$ & -0.8921 & -0.0876 & -0.1892 \\
$K=20$ & -0.4370 & 0.9088 & -0.2115 \\
$K=30$ & 0.0495 & 1.4657 & 0.0155 \\
$K=40$ & 0.3472 & 1.6589 & 0.0160 \\
\hline
\end{tabular}

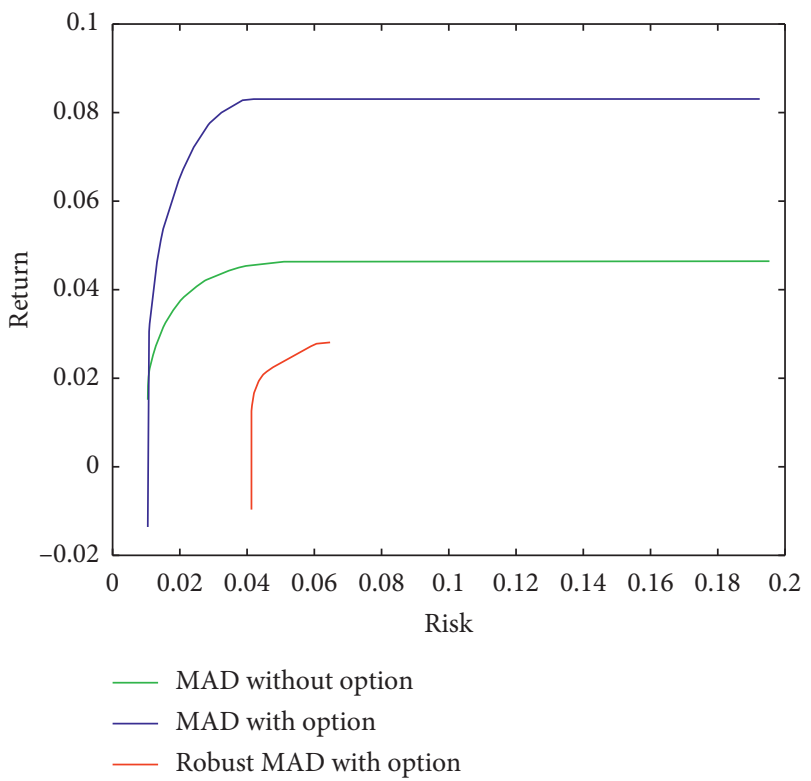

FIGURE 1: Comparison of efficient frontiers of the MAD model with and without options and its robust model for S\&P 500 index data in 2016-2018 when $K=40$ and $\lambda=0.5$.

where $\varepsilon=0.01$. To solve all models, we used CVX software using MATLAB [61].

We compare MAD model with and without options and its robust model in terms of returns and Sharpe ratios. The Sharpe ratio (SR) is calculated by

$$
\mathrm{SR}=\frac{\mu-r_{c}}{\delta}
$$

where $\mu$ is the expected portfolio return, $\delta$ is the meanabsolute deviation, and $r_{c}$ denotes risk-neutral interest rate. The results are summarized in Tables 2 and 3 for different $K$ values. As we see, portfolio with options creates significant advantage in returns and Sharpe ratios compared to the portfolio without options. Also, by comparing columns 3 and 4 of these tables, we observe that the robust model (15) can be too conservative and returns, and Sharpe ratios obtained from it, are significantly less than model (10).

Further, we compare the efficient frontiers of MAD model with and without options and its robust model (models (4), (10) and (15)) in Figure 1. As we see, the efficient frontier of the MAD model with options lies above the one without options and its robust model.

\section{Conclusions}

In this paper, we proposed extensions of the MAD model with and without options when short selling, risk-neutral interest rate, and cardinality constraint are allowed. Also, its robust model under interval uncertainty sets is given. $\mathrm{Nu}$ merical results for the S\&P 500 index showed that using options led to better performance in terms of returns and Sharpe ratios. Moreover, numerical results of the robust model showed that uncertainty may significantly reduce portfolio's returns and Sharpe ratios. Due to the importance of forecasted data and transaction costs for portfolio optimization, studying the proposed model with these factors may be considered as a future research plan.

\section{Data Availability}

The data used to support the findings of this study are available from the corresponding author upon reasonable request.

\section{Conflicts of Interest}

The authors declare that there are no conflicts of interest regarding the publication of this paper.

\section{Acknowledgments}

The second author would like to thank the Center of Excellence for Mathematical Modeling, Optimization and Combinatorial Computing (MMOCC), University of Guilan, Rasht, Iran, for partially supporting this research.

\section{References}

[1] H. Markowitz, "Portfolio selection," The Journal of Finance, vol. 7, no. 1, pp. 77-91, 1952.

[2] H. Konno and H. Yamazaki, "Mean-absolute deviation portfolio optimization model and its applications to tokyo stock market," Management Science, vol. 37, no. 5, pp. 519-531, 1991.

[3] C. D. Feinstein and M. N. Thapa, "A reformulation of a meanabsolute deviation portfolio optimization model," Management Science, vol. 39, 1993.

[4] C.-T. Chang, "A modified goal programming approach for the mean-absolute deviation portfolio optimization model," Applied Mathematics and Computation, vol. 171, no. 1, pp. 567-572, 2005.

[5] S. Gorard, "Revisiting a 90-year-old debate: the advantages of the mean deviation," British Journal of Educational Studies, vol. 53, no. 4, pp. 417-430, 2005. 
[6] G. Kasenbacher, J. Lee, and K. Euchukanonchai, Mean-variance vs. Mean-Absolute Deviation: A Performance Comparison of Portfolio Optimization Models, University of British Columbia, Vancouver, Canada, 2017.

[7] M. R. T. Baghdadabad, "An empirical analysis of funds' alternative measures in the mean absolute deviation (MAD) framework," International Journal of Emerging Markets, vol. 10, p. 726, 2015.

[8] P. Byrne and S. Lee, "Sector, region or function? a MAD reassessment of real estate diversification in great britain," Journal of Property Investment \& Finance, vol. 29, no. 2, pp. 167-189, 2011.

[9] Z. Dai, H. Zhu, and F. Wen, "Two nonparametric approaches to mean absolute deviation portfolio selection model," Journal of Industrial \& Management Optimization, vol. 16, no. 5, pp. 2283-2303, 2020.

[10] M. L. Erdaş, "Developing a portfolio optimization model based on linear programming under certain constraints: an application on borsa istanbul 30 index," Tesam Akademi, vol. 7, pp. 115-141, 2020.

[11] J. Hwang, H.-J. Kim, and J. Park, "Managing risks in an open computing environment using mean absolute deviation portfolio optimization," Future Generation Computer Systems, vol. 26, no. 8, pp. 1381-1390, 2010.

[12] C. E. Kalfin and Sukono, "Optimization of the mean-absolute deviation portfolio investment in some mining stocks using the singular covariance matrix method," Journal of Physics: Conference Series, vol. 1315, Article ID 012002, 2019.

[13] P. Li, Y. Han, and Y. Xia, "Portfolio optimization using asymmetry robust mean absolute deviation model," Finance Research Letters, vol. 18, pp. 353-362, 2016.

[14] R. Mansini and M. G. Speranza, Linear and Mixed Integer Programming for Portfolio Optimization, Springer, Berlin, Germany, 2015.

[15] G. Rehnman and N. Tesch, Application of Mean Absolute Deviation Optimization in Portfolio Management, KTH Royal Institute of Technology, Stockholm, Sweden, 2018.

[16] L. P. D. Silva, D. Alem, and F. L. D. Carvalho, "Portfolio optimization using mean absolute deviation (MAD) and conditional value-at-risk (CVaR)," Production, vol. 27, pp. 1-14, 2017.

[17] J. Lintner, "Security prices, risk, and maximal gains from diversification," The Journal of Finance, vol. 20, no. 4, pp. 587-615, 1965.

[18] Z. Dai and F. Wen, "Some improved sparse and stable portfolio optimization problems," Finance Research Letters, vol. 27, pp. 46-52, 2018.

[19] P. J. Kremer, S. Lee, M. Bogdan, and S. Paterlini, "Sparse portfolio selection via the sorted 11 -Norm," Journal of Banking \& Finance, vol. 110, Article ID 105687, 2020.

[20] A. Mousavi, M. Rezaee, and R. Ayanzadeh, "A survey on compressive sensing: classical results and recent advancements," Journal of Mathematical Modeling, vol. 8, pp. 309-344, 2020.

[21] R. H. Kwon and S. J. Stoyan, "Mean-absolute deviation portfolio models with discrete choice constraints," Algorithmic Operations Research, vol. 6, pp. 118-134, 2011.

[22] H. A. Le Thi and M. Moeini, "Long-short portfolio optimization under cardinality constraints by difference of convex functions algorithm," Journal of Optimization Theory and Applications, vol. 161, pp. 199-224, 2014.

[23] L. T. H. An and P. D. Tao, "Large-scale molecular optimization from distance matrices by a D.C. optimization approach," SIAM Journal on Optimization, vol. 14, no. 1, pp. 77-114, 2003.
[24] P. D. Tao, "Convex analysis approach to DC programming: theory, algorithms and applications," Acta Mathematica Vietnamica, vol. 22, pp. 289-355, 1997.

[25] P. D. Tao, "The DC (difference of convex functions) programming and DCA revisited with DC models of real world nonconvex optimization problems," Annals of Operations Research, vol. 133, pp. 23-46, 2005.

[26] K. P. Anagnostopoulos and G. Mamanis, “The mean-variance cardinality constrained portfolio optimization problem: an experimental evaluation of five multiobjective evolutionary algorithms," Expert Systems with Applications, vol. 38, pp. 14208-14217, 2011.

[27] J. Gao and D. Li, "Optimal cardinality constrained portfolio selection,” Operations Research, vol. 61, no. 3, pp. 745-761, 2013.

[28] F. Cesarone, A. Scozzari, and F. Tardella, "A new method for mean-variance portfolio optimization with cardinality constraints," Annals of Operations Research, vol. 205, no. 1, pp. 213-234, 2013.

[29] T. Khodamoradi, M. Salahi, and A. R. Najafi, "Cardinality constrained portfolio optimization with short selling and riskneutral interest rate," Decisions in Economics and Finance, 2020.

[30] T. Khodamoradi, M. Salahi, and A. R. Najafi, "A note on CCMV portfolio optimization model with short selling and risk-neutral interest rate," Statistics, Optimization \& Information Computing, vol. 8, pp. 0-9, 2020.

[31] X. Yu, "Robust portfolio optimization with options under ve constraint using Monte Carlo," Journal of Computers, vol. 8, pp. 1580-1587, 2013.

[32] Y. Zhu, X. Wu, I.-L. Chern, and Z.-Z. Sun, Derivative Securities and Difference Methods, Springer, Berlin, Germany, 2004.

[33] J. C. Hull, Options Futures and Other Derivatives, Pearson Education India, Bengaluru, India, 2003.

[34] N. Topaloglou, H. Vladimirou, and S. A. Zenios, "Optimizing international portfolios with options and forwards," Journal of Banking \& Finance, vol. 35, no. 12, pp. 3188-3201, 2011.

[35] M. A. Maasar, D. Roman, and P. Date, "Risk minimisation using options and risky assets," Operational Research, pp. 1-22, 2019.

[36] H. Davari-Ardakani, M. Aminnayeri, and A. Seifi, "Multistage portfolio optimization with stocks and options," International Transactions in Operational Research, vol. 23, no. 3, pp. 593-622, 2016.

[37] C. Dert and B. Oldenkamp, "Optimal guaranteed return portfolios and the casino effect," Operations Research, vol. 48, no. 5, pp. 768-775, 2000.

[38] J. Liang, S. Zhang, D. Li et al., "Optioned portfolio selection: models and analysis," Mathematical Finance, vol. 18, no. 4, pp. 569-593, 2008.

[39] F. Lutgens, J. Sturm, and A. Kolen, "Robust one-period option hedging," Operations Research, vol. 54, no. 6, pp. 1051-1062, 2006.

[40] G. Scheuenstuhl and R. Zagst, "Integrated portfolio management with options," European Journal of Operational Research, vol. 185, no. 3, pp. 1477-1500, 2008.

[41] L. Yin and L. Han, "Options strategies for international portfolios with overall risk management via multi-stage stochastic programming," Annals of Operations Research, vol. 206, no. 1, pp. 557-576, 2013.

[42] L. Zhao and D. P. Palomar, "A markowitz portfolio approach to options trading," IEEE Transactions on Signal Processing, vol. 66, no. 16, pp. 4223-4238, 2018. 
[43] Z. Dai, X. Dong, J. Kang, and L. Hong, "Forecasting stock market returns: new technical indicators and two-step economic constraint method," The North American Journal of Economics and Finance, vol. 53, p. 101216, 2020.

[44] Z. Dai, H. Zhu, and J. Kang, "New technical indicators and stock returns predictability," International Review of Economics \& Finance, vol. 71, pp. 127-142, 2020.

[45] A. L. Soyster, "Technical note-convex programming with setinclusive constraints and applications to inexact linear programming," Operations Research, vol. 21, no. 5, pp. 1154-1157, 1973.

[46] A. Ben-Tal and A. Nemirovski, "Robust solutions of linear programming problems contaminated with uncertain data," Mathematical Programming, vol. 88, no. 3, pp. 411-424, 2000.

[47] D. Bertsimas and M. Sim, "The price of robustness," Operations Research, vol. 52, no. 1, pp. 35-53, 2004.

[48] Y. Moon and T. Yao, "A robust mean absolute deviation model for portfolio optimization," Computers \& Operations Research, vol. 38, no. 9, pp. 1251-1258, 2011.

[49] S. Zymler, B. Rustem, and D. Kuhn, "Robust portfolio optimization with derivative insurance guarantees," European Journal of Operational Research, vol. 210, no. 2, pp. 410-424, 2011.

[50] V. DeMiguel and F. J. Nogales, "Portfolio selection with robust estimation," Operations Research, vol. 57, no. 3, pp. 560-577, 2009.

[51] B. Fastrich and P. Winker, "Robust portfolio optimization with a hybrid heuristic algorithm," Computational Management Science, vol. 9, no. 1, pp. 63-88, 2012.

[52] L. Garlappi, R. Uppal, and T. Wang, "Portfolio selection with parameter and model uncertainty: a multi-prior approach," Review of Financial Studies, vol. 20, no. 1, pp. 41-81, 2006.

[53] A. Ghahtarani and A. A. Najafi, "Robust goal programming for multi-objective portfolio selection problem," Economic Modelling, vol. 33, pp. 588-592, 2013.

[54] D. Goldfarb and G. Iyengar, "Robust convex quadratically constrained programs," Mathematical Programming, vol. 97, no. 3, pp. 495-515, 2003.

[55] T. Khodamoradi, M. Salahi, and A. Najafi, "Robust ccmv model with short selling and risk-neutral interest rate," Physica A: Statistical Mechanics and its Applications, vol. 547, Article ID 124429, 2020.

[56] S. Lotfi, M. Salahi, and F. Mehrdoust, "Adjusted robust meanvalue-at-risk model: less conservative robust portfolios," Optimization and Engineering, vol. 18, no. 2, pp. 467-497, 2017.

[57] S. Lotfi, M. Salahi, and F. Mehrdoust, "Robust portfolio selection with polyhedral ambiguous inputs," Journal of Mathematical Modeling, vol. 5, pp. 15-26, 2017.

[58] B. Rustem and M. Howe, Algorithms for Worst-Case Design and Applications to Risk Management, Princeton University Press, Princeton, NJ, USA, 2009.

[59] E. D. Supandi and D. Rosadi, "An empirical comparison between robust estimation and robust optimization to meanvariance portfolio," Journal of Modern Applied Statistical Methods, vol. 16, p. 32, 2017.

[60] R. H. Tütüncü and M. Koenig, "Robust asset allocation," Annals of Operations Research, vol. 132, no. 1-4, pp. 157-187, 2004.

[61] M. Grant, S. Boyd, and Y. Ye, Cvx: Matlab Software for Disciplined Convex Programming, 2014, http://cvxr.com/cvx. 